\title{
Selection of an Appropriate Basis Set for Accurate Description of Binding Energy: A First Principles Study
}

\author{
Salih AKBUDAK ${ }^{* 1}$ \\ ${ }^{1}$ Adıyaman University, Arts and Sciences Faculty, Physics Department, 02040, Adıyaman
}

(Alınış / Received: 06.06.2017, Kabul / Accepted: 26.09.2017, Online Yayınlanma / Published Online: 01.11.2017)

\section{Keywords}

Binding energy,

Correlation-Consistent basis sets,

Electronic structure methods,

Coupled Cluster Singles and Doubles

with Perturbative Triples (CCSD(T))

\begin{abstract}
Binding energies of $\mathrm{Ar}_{2}$ and $\mathrm{H}_{2}$ dimers have been investigated using correlation consistent cc-pVXZ and aug-cc-pVXZ basis sets together with Coupled Cluster with Singles and Doubles with Perturbative Triples (CCSD(T)) method. Two point extrapolations to complete basis set limit has been applied to reduce basis set incompleteness (BSIE) error. Discrepancy of our theoretical binding energy values from current experimental binding energy values in literature both for $\mathrm{Ar}_{2}$ and $\mathrm{H}_{2}$ dimers observed to be less than $1 \mathrm{kcal} / \mathrm{mol}$.
\end{abstract}

\section{Bağlanma Enerjilerinin Doğru Tanımlanması için Uygun Bir Baz Setinin Seçilmesi: Bir İlk-ilkeler Yöntemi Çalışması}

\author{
Anahtar Kelimeler \\ Bağlanma enerjisi, \\ Korelasyon-Uyumlu baz setleri, \\ Elektronik yapı metodları, \\ İkili ve üçlü pertürbatif \\ çiftlenimli küme yöntemi
}

\begin{abstract}
Özet: $\mathrm{Ar}_{2}$ and $\mathrm{H}_{2}$ dimerlerinin bağlanma enerjileri korelasyon- uyumlu ccpVXZ ve aug-cc-pVXZ baz setleri kullanılarak ikili ve üçlü pertürbatif çiftlenimli küme yöntemi ile incelendi. Baz seti noksanlık hatalarını azaltmak için tam baz limitine iki noktalı ekstrapolasyon tekniği uygulandı. Elde edilen teorik bağlanma enerjilerinin literatürdeki mevcut deneysel bağlanma enerjilerinden farkının hem $\mathrm{Ar}_{2}$ hem de $\mathrm{H}_{2}$ dimerleri için $1 \mathrm{kcal} / \mathrm{mol}$ 'den daha az olduğu gözlendi.
\end{abstract}

\section{Introduction}

Over the years there has been significant improvements in computer technology which has boosted up theoretical calculations carried out in physics, chemistry and material science and engineering [1-5]. Electronic structure calculations which try to understand the characteristics of system by solving Schrödinger equation comprehend most of the theoretical calculations in physics and chemistry [6-12]. However, exact solution of Schrödinger equation is only possible for a limited number of problems like particle in a box, hydrogen atom and harmonic oscillator. If the system of interest is composed of two and more electrons, we need to take into account electron correlations in the system which increase computation time by $\mathrm{N}^{3}$ where $\mathrm{N}$ is the number of electrons. Schrödinger equation can only be solved approximately. Density Functional Theory which is one of the distinguished electronic structure theory is implemented under different softwares like VASP, Quantum Espresso, Orca, Gaussian, Siesta i.e., [13-16]. These different softwares use different basis sets. For instance VASP and Quantum Espresso use plane-wave based basis sets [17-22], ORCA and Gaussian softwares use gaussian basis sets [23-26]. Siesta uses numeric-atom centered orbital basis sets [27-28].

In all electronic structure methods accuracy of Schrödinger equation is determined by the exhange correlation method and basis set. So we can say that there are two types of errors in electronic structure calculations. One is due to exchange correlation method and other is due to basis set. If the exchange correlation method selected for calculations is from Hartree Fock (HF) to $\operatorname{CCSD}(\mathrm{T})$ computation time increases significantly with respect to number of basis functions. So we have to find a balance between computation time and accuracy. However we should keep in mind that there are many basis sets in the literature. Among the most common basis sets are Dunning's and Huzinaga's correlation consistent basis sets [29-36], John A. Pople's basis sets [37-43], Peterson's basis sets [44-46] and Jan Erik Almlöf's basis sets [47-49]. In this paper we have used Dunning's correlation consistent basis sets together with $\operatorname{CCSD}(\mathrm{T})$ method. Since $\operatorname{CCSD}(\mathrm{T})$ is an advanced 
correlated method which reduces the error to less than $1 \mathrm{kcal} / \mathrm{mol}$, it is accepted as gold standart method among all advanced methods. So, using this method we observed the effect of cc-pVDZ, cc-pVTZ, cc-pVQZ, cc-pV5Z, aug-cc-pVDZ, aug-cc-pVTZ and aug-cc-pVQZ basis sets on binding energies of $\mathrm{Ar}_{2}$ and $\mathrm{H}_{2}$ dimers. Due to the fact that $\mathrm{Ar}$ and $\mathrm{H}$ are one of the most abundant elements in nature, we have made $\operatorname{CSSD}(\mathrm{T})$ calculations to reveal the effects of different correlation consistent basis sets on binding energies of these dimers. Accurate description of binding energies of dimers is a challenging case due to overlapping between basis function of one atom with the basis function of the other atom which causes a basis set superposition error $[50,51]$.

Binding energies of dimers and molecules have been intensively studied both experimentally and theoretically [52-68]. To mention some of these studies, Deible et al. studied the binding energy of the beryllium dimer using Quantum Monte Carlo method [64], Ian S.O Pimienta studied Ionic Dimers [52], E. Miliordos et al. carried out a benchmark study of the pi-pi binding energy in the benzene dimer [53], A.A Zadoroshnaya et al. studied ionization-induced structural changes in uracil dimers and their spectroscopic signatures [57], P. A Pieniazek et al. studied Electronic Structure of the benzene dimer cation [60], Lee T.S studied accurate $a b$ initio binding energies of alkaline earth metal clusters [61].

So we have investigated the effect of different basis sets on binding energies and reported our results at $\operatorname{CCSD}(\mathrm{T})$ level. CCSD $(\mathrm{T})$ is the gold standard method that gives the most accurate results so by doing this we tried to choose the best performing basis set that will have a low computation cost. Although computation time for dimers is not a big problem if we go beyond dimers and use more complex molecules computation time increases drastically. Thus selection of an appropriate basis set for calculations that will enable the desired accuracy can reduce computation time significantly.

\section{Material and Method}

For all calculations we used ORCA software [16]. As in all electronic structure methods, the first task is to solve electronic Schrödinger equation exactly. Timeindependent Schrödinger equation is given as;

$$
\left(\frac{-\hbar^{2}}{2 m} \nabla^{2}{ }_{i}+V(r)\right) \Psi=E \Psi
$$

To have detailed information about the system wavefunction should be exactly known. Wave function in Schrödinger equation can be written as a linear combination of basis functions;

$$
\Psi_{i}=\sum_{\mu}^{b} c_{\mu i} \Phi_{\mu}
$$

here $\Psi$ is wavefunction, $\mathrm{c}$ is a constant and $\emptyset$ is basis function. Wavefunction can be obtained selfconsistently from Equation 2. However to obtain exact value of wavefunction " $b$ " in Equation 2 should go to infinity $(\infty)$ which means that we should include infinitely many basis functions in our basis set. Unfortunately this is computationaly impractical because computation time scale as $\mathrm{N}^{3}$ where $\mathrm{N}$ is number of basis functions. Since we can not have infinitely basis functions in our basis set we always encounter a basis set incompleteness error (BSIE). One popular way to alleviate this BSIE is to use complete basis set extrapolation techniques. In this paper we used Halkier's two point extrapolation technique [70]. Two point extrapolation scheme is given as;

$$
\begin{aligned}
& E[X]=E[\infty]+\frac{A}{X^{3}} \\
& E[Y]=E[\infty]+\frac{A}{Y^{3}}
\end{aligned}
$$

using Equations 3 and 4 real equation of CBS can be derived as;

$$
C B S=E_{X Y}[\infty]=\frac{E[X] X^{3}-E[Y] Y^{3}}{X^{3}-Y^{3}}
$$

where $\mathrm{X}$ and $\mathrm{Y}$ are maximum angular momentum numbers in basis set. For example if we do extrapolations from $\mathrm{DZ}$ to $\mathrm{TZ}$, we take $\mathrm{X}$ as 3 and $\mathrm{Y}$ as 2.

The most important term in electronic Schrödinger equation is exchange correlation term since it can not be determined analytically for systems having two and more electrons. So, one has to apply an approximation method. In this paper, we have choosen Coupled Cluster Singles and Doubles with Perturbative Triples method which is a post-HarteeFock method. When applying this method to medium sized molecules results are satisfying. After obtaining $\operatorname{CCSD}(\mathrm{T})$ total energies for the system we have obtained binding energies of $\mathrm{H}_{2}$ and $\mathrm{Ar}_{2}$ dimers using conventional binding energy fomula;

$$
\Delta \mathrm{E}_{\text {binding }}=\mathrm{E}_{\text {molecule }}-2 \mathrm{E}_{\text {atom }}
$$

where $\mathrm{E}$ is the total energy )[69].

\section{Results}

Figure 1 (a)-(e) shows the absolute binding energy values with respect to cc-pVXZ (X=D, T, Q and 5) and aug-cc-pVXZ (X=D, T and $Q$ ) basis sets for $\mathrm{Ar}_{2}$ dimer. All electronic structure calculations are carried out at $\operatorname{CCSD}(\mathrm{T})$ level. Extrapolated binding energies for Figures 1(a)-(e) are 0.0049, 0.0054, 0.005, 0.0067 and $0.0064 \mathrm{eV}$ respectively. 


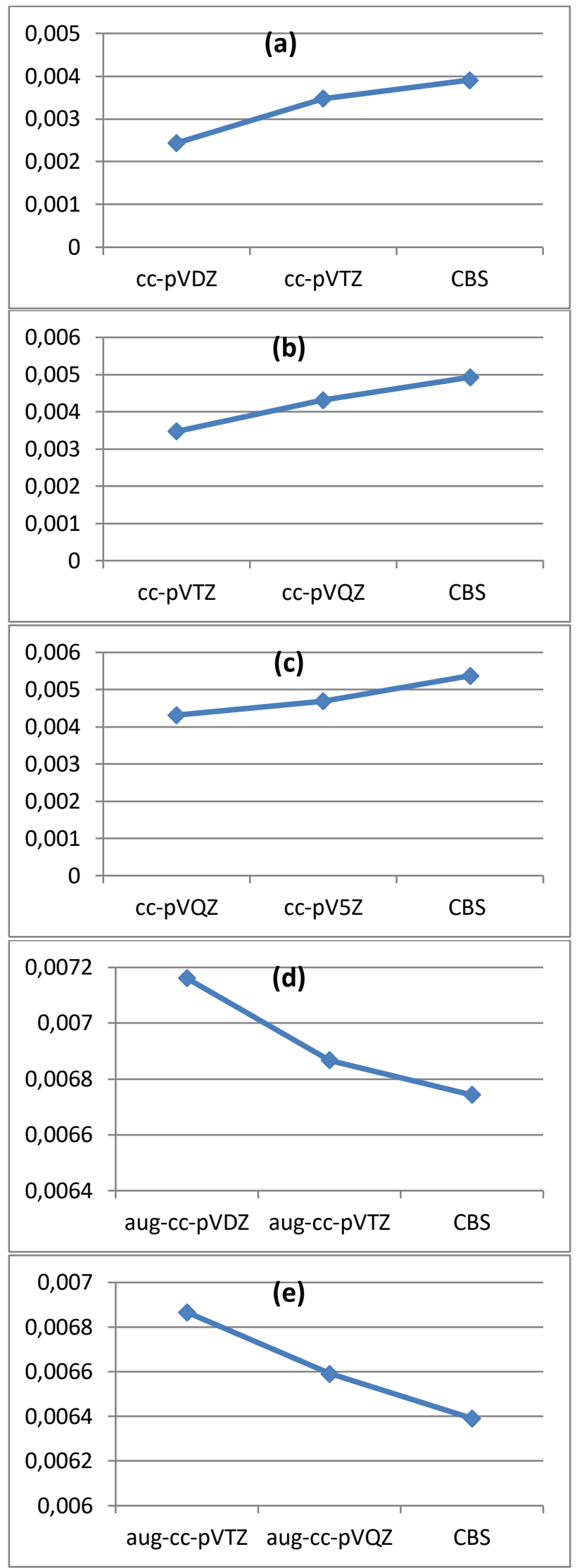

Figure 1. Absolute binding energy (eV) of Ar2 dimer with respect to cc-pVDZ and cc-pVTZ in Figure 1(a), cc-pVTZ and cc-pVQZ in Figure 1(b), cc-pVQZ and cc-pV5Z in Figure 1(c), aug-cc-pVDZ and aug-cc-pVTZ in Figure 1(d), aug-cc-pVTZ and aug-cc-pVQZ in Figure 1(e). Extrapolation to CBS limit is from DZ to TZ, TZ to QZ, QZ to $5 Z$ for 1 (a)-(c) and from DZ to $3 Z$ and $3 Z$ to $Q Z$ for aug-cc-pVTZ and aug-cc-pVQZ for $1(\mathrm{~d})-(\mathrm{e})$.
Figure 2 shows the binding energies of $\mathrm{Ar}_{2}$ with respect to correlation consistent cc-pVDZ, cc-pVTZ, cc-pVQZ, cc-pV5Z, aug-cc-pVDZ, aug-cc-pVTZ and aug-cc-pVQZ basis sets. Binding energies obtained with these basis sets are 0.0024, 0.003.5, 0.0043, $0.0047,0.0072,0.0069$ and $0.006 .6 \mathrm{eV}$ respectively. From these observations the best available basis sets for description of binding energy of $\mathrm{Ar}_{2}$ are cc-pVTZ and cc-pVQZ since energy discrepancy from aug-ccpVQZ is less than $2 \mathrm{meV}$. Computationaly the most demanding basis set is aug-cc-pVQZ. If cc-pVTZ and cc-pVQZ basis sets are used, and $3 Z$ to QZ two point extrapolation is applied, computation time decreases significantly compared to basis sets used in figures 1 (c)-(d). Results obtained with this basis sets are in chemical accuracy limit ( $1 \mathrm{kcal} / \mathrm{mol}$ or $43 \mathrm{meV}$ ) since experimental binding energy of $\mathrm{Ar}_{2}$ dimer is $12 \mathrm{meV}$ $[65,71]$.

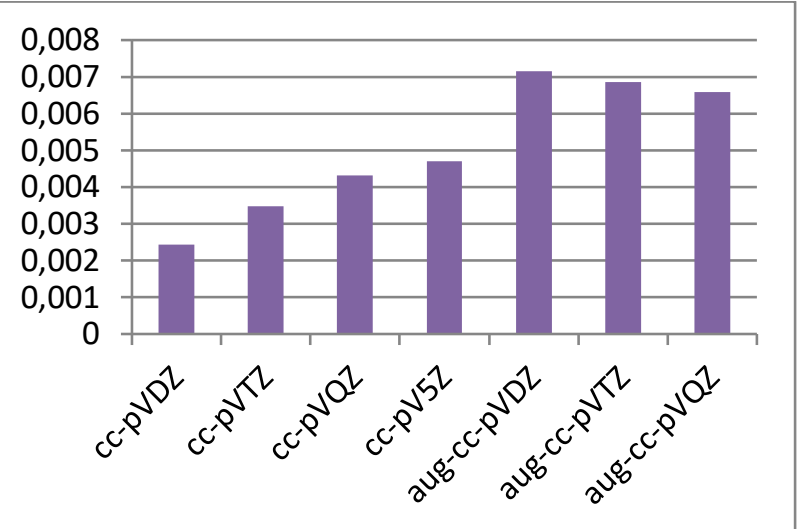

Figure 2. Absolute binding energy $(\mathrm{eV})$ of $\mathrm{Ar}_{2}$ dimer with respect to cc-pVXZ (X=D, T, Q 5) and aug-cc-pVXZ (X=D, T, Q) basis sets.

Figures 3 (a)-(e) show the absolute binding energy values of $\mathrm{H}_{2}$ dimer with respect to different correlation consistent basis sets of Dunning [29]. From Figures 3 (a) to (e) CBS extrapolated values are obtained by applying DZ to $3 Z, 3 Z$ to $\mathrm{QZ}, \mathrm{QZ}$ to $5 \mathrm{Z}$ two point extrapolations for cc-pVXZ (X=D, T, Q 5) and DZ, TZ and QZ extrapolations for aug-cc-pVXZ $(X=D, T$, Q) basis sets. Extrapolated binding energy values shown by CBS in Figures 3 (a)-(e) are 4. 39, 4. 01, 3. $41,3.29$ and $3.26 \mathrm{eV}$ respectively.

Figure 4 shows binding energy of $\mathrm{H}_{2}$ dimer for ccpVDZ, cc-pVTZ, cc-pVQZ, cc-pV5Z, aug-cc-pVDZ, augcc-pVTZ and aug-cc-pVQZ basis sets at CCSD(T) level. Binding energies calculated are 6. 09, 4. 89, 4. 38, 3. 91, 3. $17 \mathrm{eV}$ for cc-pVXZ (X=D, T, Q 5) basis sets and 3. $25 \mathrm{eV}$ for aug-cc-pVXZ(X=D, T, Q) basis sets. Experimental binding energy of $\mathrm{H}_{2}$ dimer is 4.40 eV[71] so if we choose cc-pVDZ and cc-pVTZ basis sets and make extrapolations to complete basis set limit (CBS) from DZ to TZ we obtain a binding energy of $4,39 \mathrm{eV}$ for $\mathrm{H}_{2}$. So theoretical value we obtained at $\operatorname{CCSD}(\mathrm{T})$ level is $0,01 \mathrm{eV}$ less than experimental value. And we can also use cc-pVTZ basis sets which has a binding energy of $4,38 \mathrm{eV}$. If we use this basis set we do not need to make extrapolations. 

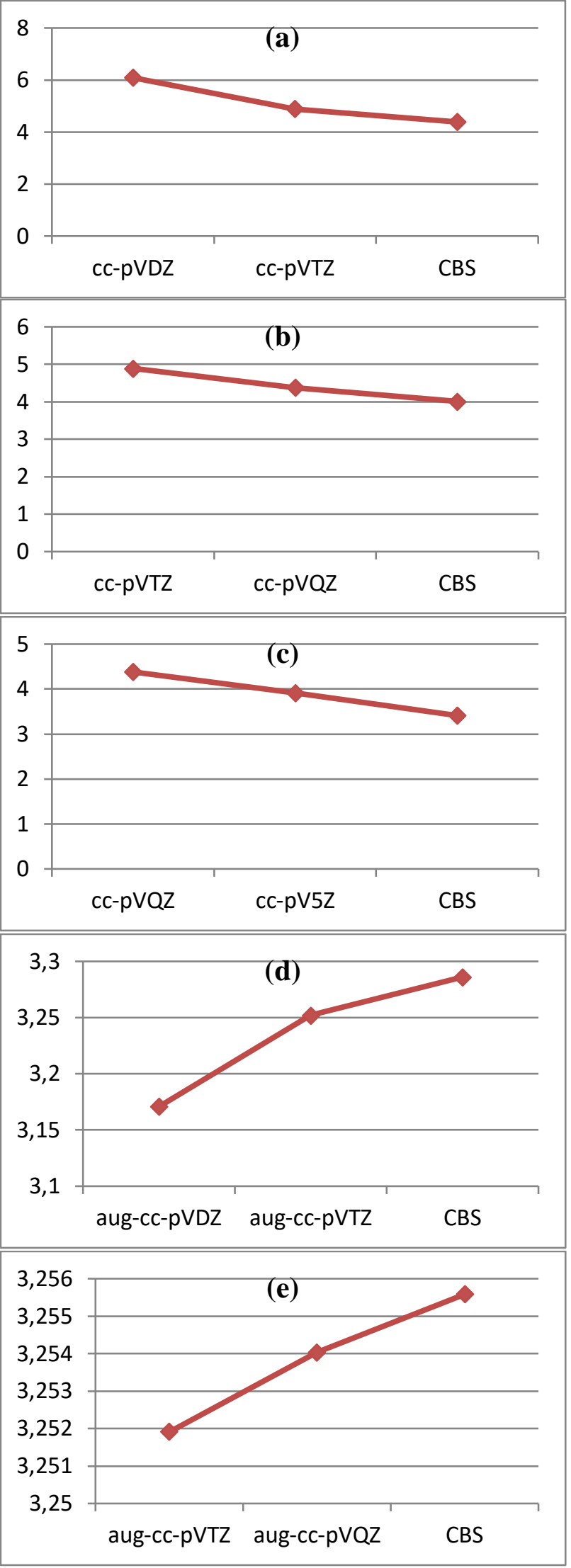

Figure 3. Absolute binding energy $(\mathrm{eV})$ of $\mathrm{H}_{2}$ dimer with respect to cc-pVDZ and cc-pVTZ in Figure 3(a), cc-pVTZ and cc-pVQZ in Figure 3(b), cc-pVQZ and cc-pV5Z in Figure 3(c), aug-cc-pVDZ and aug-cc-pVTZ in Figure 3(d), aug-ccpVTZ and aug-cc-pVQZ in Figure 3(e). Extrapolation to CBS limit is from DZ to TZ, TZ to QZ, QZ to $5 Z$ for 3(a)-(c) and from DZ to $3 \mathrm{Z}$ and $3 \mathrm{Z}$ to $\mathrm{QZ}$ for aug-cc-pVTZ and aug-ccpVQZ for 3(d)-(e).

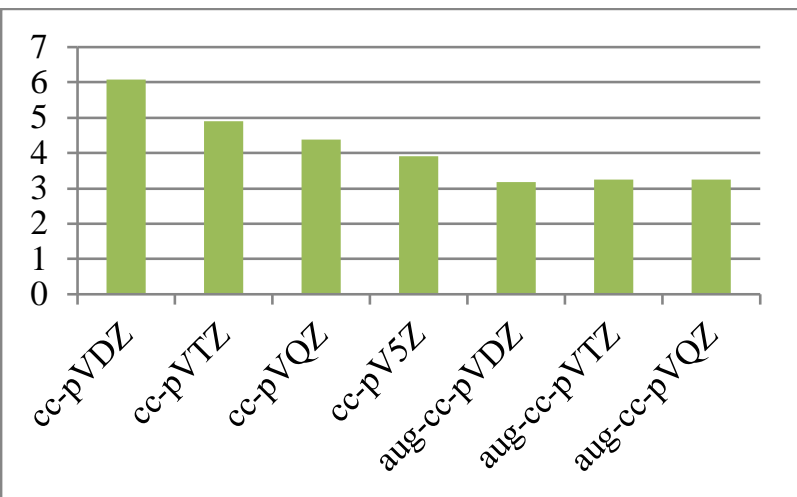

Figure 4. Absolute binding energy $(\mathrm{eV})$ of $\mathrm{H}_{2}$ dimer with respect to cc-pVXZ $(X=D, T, Q, 5)$ and aug-cc-pVXZ $(X=D, T$, Q).

\section{Discussion and Conclusion}

In summary, we have investigated the effect of different basis sets on binding energies and tried to find the best basis set for each dimer in terms of computation time and accuracy. In principle what we expect for a specific calculation is that the larger is the basis set we use the higher the accurate results we have. But, especially for large molecules, not for dimers computation time is also very important, because when deciding to use a basis set, computation time is an important constraint, so selection of a basis set of modest size that would give the desired accuracy for calculation is the main objective in electronic structure calculations. The need for these types of studies is indispensable since there is a huge number of basis sets in literature and they are growing exponentially. So, it is always a need to compare the performance of existing basis sets for a system and find the best performing basis set in terms of computation time and accuracy. And we believe that this paper will initiate more studies about different molecules and dimers that will shed light to computational chemists and computational physicists. The investigation of large molecules with different basis sets will be carried out in the future.

\section{References}

[1] Shi, H. L., Pan, H., Zhang, Y. W., Yakobson, B. I. 2013. Quasiparticle band structures and optical properties of strained monolayer MoS2 and WS2. Physical Review B, 87(2013), 155304.

[2] Moreels, I., Lambert, K. D., Muyncuk Smeets, D., Nollet, T., Martins, J. C., Vanhaecke, F., Vantomme, A., Delerue, C., Allan, G., Hens, Z. 2009. Size-Dependent Optical Properties of Colloidal $\mathrm{PbS}$ Quantum Dots. ACS NANO, 3(2009), 3023.

[3] Nazarian, D., Camp, J.S, Chung, Y.G, Snurr, R.Q., Sholl, D.S. 2017. Large-Scale Refinement of Metal-Organic Framework Structures Using Density Functional Theory. Chemistry of Materials, 29(2017), 2521-2528. 
[4] Yoffe, A.D. 2001. Semiconductor quantum dots and related systems: Electronic, optical, luminescence and related properties of low dimensional systems. Advances in Physics. 50(2001), 1-208.

[5] Boukhvalov, D. W., Katsnelson, M. I.; Lichtenstein, A. I. 2008. Hydrogen on graphene: Electronic structure, total energy, structural distortions, and magnetism from first-principles calculations. Physical Review B, 77(2008), 035427.

[6] Gece, G. 2008. The use of quantum chemical methods in corrosion inhibitor studies. Corrosion Science 50 (2008), 2981-2992.

[7] Gece, G., Bigiç, S. 2010. A theoretical study on the inhibition efficiencies of some amino acids as corrosion inhibitors of nickel. Corrosion Science 55(2010), 3304-3308.

[8] Mete, E., Yilmaz, A., Danışman, M. F. 2016. A van der Waals density functional investigation of carboranethiol self-assembled monolayers on $\mathrm{Au}$ (111). Physical Chemistry Chemical Physics, 18(2016), 12920-12927.

[9] Onida, G., Reining, L., Rubio, A. 2002. Electronic excitations: density-functional versus manybody Green's-function approaches. Review of Modern Physics, 74(2002), 601.

[10] Marzari, N., Vanderbilt, D. 1997. Maximally localized generalized Wannier functions for composite energy bands. Physical Review B, 57(1997), 12845.

[11] Tkatchenko, A., Scheffler, M. 2009. Accurate molecular van der Waals interactions from ground-state electron density and free-atom reference data. Physical Review Letters, 102(2009), 073005.

[12] Karakaya, M., Sert, Y., Kurekci, M., Eskiyurt, B., Cirak, C. 2015. Theoretical and experimental investigations on vibrational and structural properties of tolazamide. Journal of Molecular Structure 1095(2015), 87-95.

[13] Kresse, G., Furthmuller, J. 1996. Efficient iterative schemes for ab initio total-energy calculations using a plane-wave basis set. Physical Review B, 54(1996), 11169.

[14] Giannozzi, P., Baroni, S., Bonini, N., Calandra, M., Car, R., Cavazzoni, C., Ceresoli, D., Chiarotti, G. L., Cococ cioni, M., Dabo, I., Dol Corso, A., De Groncoli, S., Fabris, S., Fratesi, G., Gebauer, R., Gerstmann, U., Gou goussis, C., Kokalj, A., Lazzeri, M., Martin-Samos, L., Marzari, N., Mauri, F., Mazzarello, R., Paolini, S., Pasquarello, A., Paulatto, L., Sbraccia, C., Seitsonen, A. P., Smogunov, A., Umari, P., Wentzcovitch, R. M. 2009. QUANTUM ESPRESSO: a modular and open-source software project for quantum simulation of materials. Journal of Physics Condensed Matter, 21(2009), 395502.

[15] Soler, J. M., Artacho, E., Gale, J. D., Garcia, A., Unquera, J. J., Ordejon, P., Sanchez-Portal, D. 2002. The SIESTA method for ab initio order- N materials simulation. Journal of Physics Condensed Matter, 14(2002), 2745-2779.

[16] Neese, F. 2012. The ORCA program system. Wires Compuational Molecular Science, 2(2012), 73-78.

[17] Hafner, J. 2008. Ab-initio simulations of materials using VASP: Density-functional theory and beyond. Journal of Compuational Chemistry, 29(2008), 2044-2078.

[18] Setyawan, W., 2010. Curtarolo, S. Highthroughput electronic band structure calculations: Challenges and and tools Compuational Material Science, 49(2010), 299312.

[19] Ugeda, M. M., Brihuega, F., Guinea, J. M., GomezRodriguez. 10. Missing Atom as a Source of Carbon Magnetism. Physical Review Letters, 104(2010), 096804.

[20] A. Tkatchenko, A., Jr. DiStasio, R. A., Car, R., Scheffler, M. 2012. Accurate and efficient method for many-body van der Waals interactions. Physical Review Letters, 108(2012), 236402.

[21] Zahid, F., Lake, R. R. 2010. Thermoelectric properties of Bi2Te3 atomic quintuple thin films. Applied Physics Letters, 97(2010), 212102.

[22] P. Rani, P., Jindal, V. K. 2013. Designing band gap of graphene by $\mathrm{B}$ and $\mathrm{N}$ dopant atoms, RCS Advances, 3(2013), 802-812.

[23] Neese, F., Wennmohs, F., Hansen, A., Becker, U. 2009. Efficient, approximate and parallel Hartree-Fock and hybrid DFT calculations. A 'chain-of-spheres' algorithm for Hartree-Fock exchange. Chemical Phyics, 356(2009), 98-109.

[24] Neese, F. J. 2007. Calculation of the zero-field splitting tensor on the basis of hybrid density functional and Hartree-Fock theory. Chemical Physics, 127(2007), 164112.

[25] Neese, F. 2003. A spectroscopy oriented configuration interaction procedure. Journal of Chemical Physics, 119(2003), 9428.

[26] Dunning, T. H. 2000. A Road Map for the Calculation of Molecular Binding Energies. Journal of Physical Chemistry A, 104(2000), 9062-9080.

[27] Tsolakidis, A., Sanchez-Portal, D., Martin, R. M. 2002. Calculation of the optical response of atomic clusters using time-dependent density functional theory and local orbitals. Physical Review B, 66(2002), 235416. 
[28] Wahnon, P., Tablero, C. 2002. Ab initio electronic structure calculations for metallic intermediate band formation in photovoltaic materials. Physical Review B 65(2002), 165115.

[29] Dunning, T. H. 1989. Gaussian basis sets for use in correlated molecular calculations. I. The atoms boron through neon and hydrogen. Journal of Chemical Phyics, 90(1989), 10071023.

[30] Kendall, R. A., Dunning, T. H., Harrison, R. 1992. Electron affinities of the first-row atoms revisited. Systematic basis sets and wave functions. Journal of Chemical Physics, 1992, 96(1992), 6796.

[31] Dunning, T. H. 1970. Gaussian Basis Functions for Use in Molecular Calculations. I. Contraction of (9s5p) Atomic Basis Sets for First-Row Atoms. The Journal of Chemical Physics, 53(1970), 2823.

[32] Dunning, T. H. 1971. Gaussian basis functions for use in molecular calculations. III. Contraction of (10s6p) atomic basis sets for the first-row atoms. Journal of Chemical Physics, 55(1971), 716-723.

[33] Dunning, T. H., Peterson, K. A., Wilson, A. K. 2001. Gaussian basis sets for use in correlated molecular calculations. X. The atoms aluminum through argon revisited. Journal of Chemical Physics, 2001, 114(2001), 9244.

[34] Woon, D. E., Dunning, T. H. 1993. Calculation of the electron affinities of the second row atoms: Al-Cl. Journal of Chemical Physics, 99(1993), 3730-3737.

[35] Woon, D. E., Dunning, T. H. 1993. Gaussian basis sets for use in correlated molecular calculations. III. The atoms aluminum through argon. Journal of Chemical Physics, 98(1993), 1358-1371.

[36] Peterson, K. A., Dunning, T. H. 2002. Accurate correlation consistent basis sets for molecular core-valence correlation effects: The second row atoms $\mathrm{Al}-\mathrm{Ar}$, and the first row atoms $\mathrm{B}-\mathrm{Ne}$ revisited. Journal of Chemical Physics, 117(2002), 10548-10560.

[37] Hehre, W. J., Stewart, R. F., Pople, J. A. 1969. SelfConsistent Molecular-Orbital Methods. I. Use of Gaussian Expansions of Slater-Type Atomic Orbitals. Journal of Chemical Physics, 51(1969), 2657.

[38] Hehre, W. J., Ditchfield, R., Stewart, R. F.; Pople, J. A. 1970. Self-Consistent Molecular Orbital Methods. IV. Use of Gaussian Expansions of Slater-Type Orbitals. Extension to Second-Row Molecules. Journal of Chemical Physics, 52(1970), 2769.

[39] Binkley, J. S., Pople, J. A., Hehre, W. J. 1980. Selfconsistent molecular orbital methods. 21. Small split-valence basis sets for first-row elements.
Journal of American Chemical Society, 102(1980), 939-947.

[40] Gordon, M. S., Binkley, J. S., Pople, J. A., Pietro, W. J., Hehre, W. J. 1982. Self-consistent molecularorbital methods. 22. Small split-valence basis sets for second-row elements. Journal of American Chemical Society, 104(1982), 27972803.

[41] Hehre, W. J., Ditchfield, R., Pople, J. A. 1972. Self-Consistent Molecular Orbital Methods. XII. Further Extensions of Gaussian-Type Basis Sets for Use in Molecular Orbital Studies of Organic Molecules. Journal of Chemical Physics, 56(1972), 2257.

[42] Krishnan, R., Binkley, J. S., Seeger, R., Pople, J. A. 1980. Self-consistent molecular orbital methods. XX. A basis set for correlated wave functions. Journal of Chemical Physics. 72(1980), 650.

[43] Hariharan, P. C., Pople, J. A. 1973. The influence of polarization functions on molecular orbital hydrogenation energies. Theoretical Chimimica Acta, 28(1973), 213-222.

[44] Balabanov, N. B., Peterson, K. A. 2005. Systematically convergent basis sets for transition metals. I. All-electron correlation consistent basis sets for the 3d elements Sc-Zn. Journal of Chemical Physics, 123(2005), 064107.

[45] Peterson, K. A. 2003. Systematically convergent basis sets with relativistic pseudopotentials. I. Correlation consistent basis sets for the post-d group 13-15 elements. Journal of Chemical Physics, 119(2003), 11099-11112.

[46] Feller, D., Peterson, K. A. 1998. An examination of intrinsic errors in electronic structure methods using the EMSL computational results database and the G2 set. Journal of Chemical Physics. 108(1998), 154.

[47] Almlöf, J., Taylor, P. R. 1987. General contraction of Gaussian basis sets. I. Atomic natural orbitals for first- and second-row atoms. Journal of Chemical Physics. 86(1987), 4070.

[48] Almlöf, J., Taylor, P. R. 1990. General contraction of Gaussian basis sets. II. Atomic natural orbitals and the calculation of atomic and molecular properties. Journal of Chemical Physics, 92(1990), 551.

[49] Almlöf, J. 1991. Atomic natural orbital (ANO) basis sets for quantum chemical calculations. Advances Quantum Chemistry, 22(1991), 301373.

[50] Simon, S., Duran, M., Dannenberg, J. J. 1996. How does basis set superposition error change the potential surfaces for hydrogen-bonded dimers?. Journal of Chemical Physics, 105(1996), 11024.

[51] Antony, J., Grimme, S. 2006. Density functional theory including dispersion corrections for intermolecular interactions in a large 
benchmark set of biologically relevant molecules. Physical Chemistry Chemical Physics, 8(2006), 5287-5293.

[52] Pimienta Ian, S. O. 2015. Computational Study of Monosubstituted Azo(tetrazolepentazolium)Based Ionic Dimers. Journal of Physical Chemistry A, 119(2015), 5826-5841.

[53] Miliordos, E., Apra, E., Xantheas, S. S. 2014. Benchmark theoretical study of the $\pi-\pi$ binding energy in the benzene dimer. Journal of Physical Chemistry A, 118(2014), 7568-7578.

[54] Johll, H., Tok, E. S., Kang, H. C. 2011. Structure and properties of pure and mixed transition metal dimers on graphene. International Journal of Nanotechnology, 8(2011), 825.

[55] Song, X. D., Zhao, Y. F., Zhang, G. H., Zhang, P. X. 2011. Theoretical study on structures, binding energies and vibrational spectra of $\mathrm{M}+(\mathrm{H} 20) 2 \mathrm{Ar}(\mathrm{M}=\mathrm{Cu}, \mathrm{Ag}, \mathrm{Au})$. Computational and Theoretical Chemistry 2011, 963, 290-293.

[56] Kreutzer, J., Blaha, P., Schubert, U. 2016. Assessment of different basis sets and DFT functionals for the calculation of structural parameters, vibrational modes and ligand binding energies of $\mathrm{Zr} 402$ (carboxylate) 12 clusters. Computational and Theoretical Chemistry, 1084(2016), 162-168.

[57] Zadorozhnaya, A. A., Krylov, A. I. 2010. Ionization-Induced Structural Changes in Uracil Dimers and Their Spectroscopic Signatures. Journal of Chemical Theory and Compution, 6(2010), 705-717.

[58] Yousaf, K. E., Brothers, E. N. 2010. Applications of Screened Hybrid Density Functionals with Empirical Dispersion Corrections to Rare Gas Dimers and Solids. Journal of Chemical Theory and Compution, 8(2010), 864-872.

[59] Johll, H., Kang, H. C., Tok, E. S. 2009. Density functional theory study of $\mathrm{Fe}, \mathrm{Co}$, and $\mathrm{Ni}$ adatoms and dimers adsorbed on graphene. Physical Review B, 79(2009), 245416.

[60] Pieniazek, P. A., Krylov, A. I., Bradforth, S. E. 2007. Electronic structure of the benzene dimer cation. Journal of Chemical Physics, 127(2007), 044317.

[61] Lee, J. S. 2005. Accurate ab initio binding energies of alkaline earth metal clusters Journal of Physical Chemistry A, 109(2005), 1192711932.

[62] Sinnokrot, M. O., Valeev, E. F., Sherrill, C. D. 2002. Estimates of the ab initio limit for $\pi-\pi$ interactions: the benzene dimer. Journal of American Chemical Society, 124(2002), 10887.

[63] Tuma, C., Boese, A. D., Handy, N. C. 1999. Predicting the binding energies of $\mathrm{H}$-bonded complexes: A comparative DFT study. Physical Chemistry Chemical Physics, 1(1999), 3939.
[64] Deible, M. J., Kessler, M., Gasperich, K. E., Jordan, K. D. 2015. Quantum Monte Carlo calculation of the binding energy of the beryllium dimer. Journal of Chemical Physics, 143(2015), 084116.

[65] Elm, J., Kristensen, K. 2017. Basis set convergence of the binding energies of strongly hydrogen-bonded atmospheric clusters. 19(2017), 1122-1133.

[66] Olsson, M. A., Ryde, U. 2017. Comparison of QM/MM methods to obtain ligand-binding free energies. Journal of Chemical Theory and Computation, 13(2017), 2245-2253.

[67] Boschen, J. S., Theis, D., Ruedenberg, K., Windus, T. L . 2017. Correlation energy extrapolation by many-body expansion. The Journal of Physical Chemistry A, 121(2017), 836-844.

[68] Schütz, M., Maschio, L., Karttunen, A. J., Usvyat, D. 2017. Exfoliation Energy of Black Phosphorus Revisited: A Coupled Cluster Benchmark. Journal of Physical Chemistry Letters, 8(2017), 12901294.

[69] Ruzzinsky, A., Perdew, J. P., Gabor, C. I. 2005. Binding energy curves from nonempirical density functionals II. van der Waals bonds in rare-gas and alkaline-earth diatomics. Journal of Physical Chemistry A, 109(2005), 11015-21.

[70] Halkier, A., Helgaker, T., Jorgensen, P., Klopper, W., Olsen, K. H. J., Wilson, A. K. 1998. Basis-set convergence in correlated calculations on $\mathrm{Ne}, \mathrm{N}$, and $\mathrm{H}_{2} \mathrm{O}$. Chemical Physics Letters, 286(1998), 243-252.

[71] Lide, D. R. (ed.). 1998. Chemical Rubber Company handbook of chemistry and physics, CRC Press, Boca Raton, Florida, USA, 79th edition. 